International Journal of Production Research (2006), Vol. 44, No. 3, 545-567

DOI: 10.1080/00207540500270364

\title{
Operational Level-Based Policies in Production Rate Control of Unreliable Manufacturing Systems With Setups
}

\author{
A. Gharbi*, J.-P. Kenné** and A. Hajji* \\ * Automated Production Engineering Department, Production Systems Design and \\ Control Laboratory \\ ** Mechanical Engineering Department \\ Université du Québec, École de Technologie Supérieure, 1100, Notre Dame Street West, \\ Montreal (Quebec), Canada, H3C 1K3
}

\begin{abstract}
This paper deals with the control of the production rates and setup actions of an unreliable multiple-machine, multiple-product manufacturing system. Each part type can be processed for a specified length of time on one of the involved machine. When switching the production from one type to another, each machine requires both setup time and setup cost. Our objective is to determine the production rates and a sequence of setups in order to minimize the total setup and surplus cost. Aiming the fact that an analytical or even a numerical solution of the problem is very difficult to find, a combined approach is presented. The proposed approach is based on stochastic optimal control theory, discrete event simulation, experimental design, and response surface methodology. We will prove experimentally that an extended version of the hedging corridor policy is more realistic and guarantees better performance for two cases of study. The first one consists on the unreliable one machine case facing exponential failure and repair time distribution. The second one, which is more complex and where the optimal control theory may not be easily used to obtain the optimal control policy, consists on five machines facing non exponential failure and repair time distributions. To illustrate the contribution of the paper and the robustness of the obtained control policy, numerical examples and sensitivity analysis are presented.
\end{abstract}

Keywords: Production rates, setup policy, stochastic dynamic programming, numerical methods, simulation, experimental design.

\section{Introduction}

An important class of stochastic manufacturing systems involves non-flexible machines characterized by significant setup time and costs incurred when production is switched from one product type to another. This class of systems belongs to manufacturing systems for which the problem of determining optimal production policies have been considered by many authors. A significant portion of this research is based on the 
pioneering work of Kimemia and Gershwin (1983), who suggested a feedback formulation of the control problem in a dynamic manufacturing environment, and showed that the optimal control has a special structure called the Hedging Point Policy (HPP). For such a policy, a non-negative production surplus of parts, corresponding to optimal inventory levels, is maintained during times of excess capacity in order to hedge against future capacity shortages caused by machine failures.

For large-scale manufacturing systems (i.e., involving multiple parts and/or multiple machines), different classes of systems have been investigated in several works. An explicit formulation of the optimal control problem for an unreliable flexible machine which produces multiple part types is provided in Sethi and Zhang (1999). In addition, Gharbi and Kenné (2003) provided a sub-optimal control policy for the multiple parts multiple machines problem. The assumption made in the aforementioned classes of systems is that the machines are completely flexible, and thus do not require setup time or cost when production is switched from one part type to another.

Stochastic manufacturing systems with setup costs and/or times have been considered by Sethi and Zhang (1994), Yan and Zhang (1997) and Boukas and Kenné (1997). The proposed models lead to the optimality conditions described by the Hamilton Jacobi Bellman equations (HJB). Such equations are difficult to resolve analytically for more general cases. An explicit solution for such equations was obtained by Akella and Kumar (1986) for a one-machine, one-product manufacturing system. Numerical methods based on the Kushner approach (see Kushner and Dupuis (1992)) were used by Yan and Zhang (1997) and Boukas and Kenné (1997) for a one-machine, two-product manufacturing system. They were able to develop near-optimal control policies for production, maintenance (in Boukas and Kenné (1997)) and setup scheduling in the case of a homogeneous and machine age-dependent Markovian process, respectively.

For the one machine two products case, Yan and Zhang (1997) provide a characterization of the optimal production and setup policy by four exclusive regions as a main result. Under different assumptions, Liberopoulos and Caramanis (1997) also investigate several numerical examples so as to characterize the production and setup policies of the problem. Their results outline important properties of the value function, and those of the optimal control policy, but the structure of such a policy in the overall sample space is yet 
to be described or quantified. In the same direction, Bai and Elhafsi (1997) focused their contribution on providing a suitable production and setup policy structure, and obtained the so-called Hedging Corridor Policy (HCP). The corridor in such a policy guides the surplus trajectory to target positive stock thresholds built up in order to hedge against future capacity shortages caused by machine failures and large setup times. The setup policy in sample space quadrants related to backlog situations is still unknown with the HCP.

This paper's main contribution lies in the development of a production and setup policy for unreliable multiple-machine multiple-part type manufacturing system, for which the production and setup policy is known across the sample space. The resultant control policy, called the Modified Hedging Corridor Policy (MHCP) is more realistic and useful in the context of the production planning of manufacturing systems with setup. This paper's contribution is further illustrated by the fact that the proposed MHCP guarantees a lower incurred cost compared to that resulting from the HCP. A simulation-based experimental design approach is combined with the control theory to develop a systematic control approach, as in Gharbi and Kenné (2003), in the case of manufacturing systems involving setup. Once the superiority of the MHCP is proven through such an approach, extension to cover more complex manufacturing systems will be presented (i.e., multiple- machine multiple part type, non-exponential failure and repair time distributions), where the optimal control theory may not be easily used to obtain the control policy.

The proposed control approach consists of estimating the relationship between the incurred cost and the parameters of the control policy considered here as control factors. The Modified Hedging Corridor Policy, parameterized by these factors, is used to conduct simulation experiments. For each configuration of input factor values, the simulation model is used to determine the related output or cost incurred. An input-output data set is then generated through the simulation model. The experimental design is used to determine significant factors and/or their interactions, and the response surface methodology is applied to the input-output data obtained in order to estimate the cost function and the related optimum. Details on the combination of analytical approaches 
and simulation-based statistical methods can be found in Gharbi and Kenné (2003) and in the references they provide.

This paper is organized as follows: Section 2 presents the statement of the optimal production and setup-scheduling problem. The numerical approach and the related control policy are presented in section 3. Sections 4 and 5 describe the combined control approach and the simulation model. Section 6 outlines the experimental design approach and the response surface methodology. The usefulness of the proposed control policy and its extension to the multiple machine case with non-exponential failure and repair time distributions is presented in section 7 . The paper is concluded in section 8 .

\section{Problem Statement}

The manufacturing system under study (Figure 1) consists of $m$ unreliable machines $M_{i}$, $\mathrm{i}=1, \ldots, \mathrm{m}$ capable of producing $n$ different part types $P_{j}, \mathrm{j}=1, \ldots, \mathrm{n}$. Machines are not completely flexible in the sense that changes over time (setup activities) between part types are not easily achieved. This setup involves both time and cost to switch from the production of $P_{i}$ to $P_{j}$, denoted by $\Theta_{i j}^{k}$ and $K_{i j}^{k}$, respectively with $\mathrm{i} \neq \mathrm{j}, \mathrm{k}=1 \ldots \mathrm{m}$.

Figure 1 illustrates the system under study, its dynamic and the associated costs to be minimized.

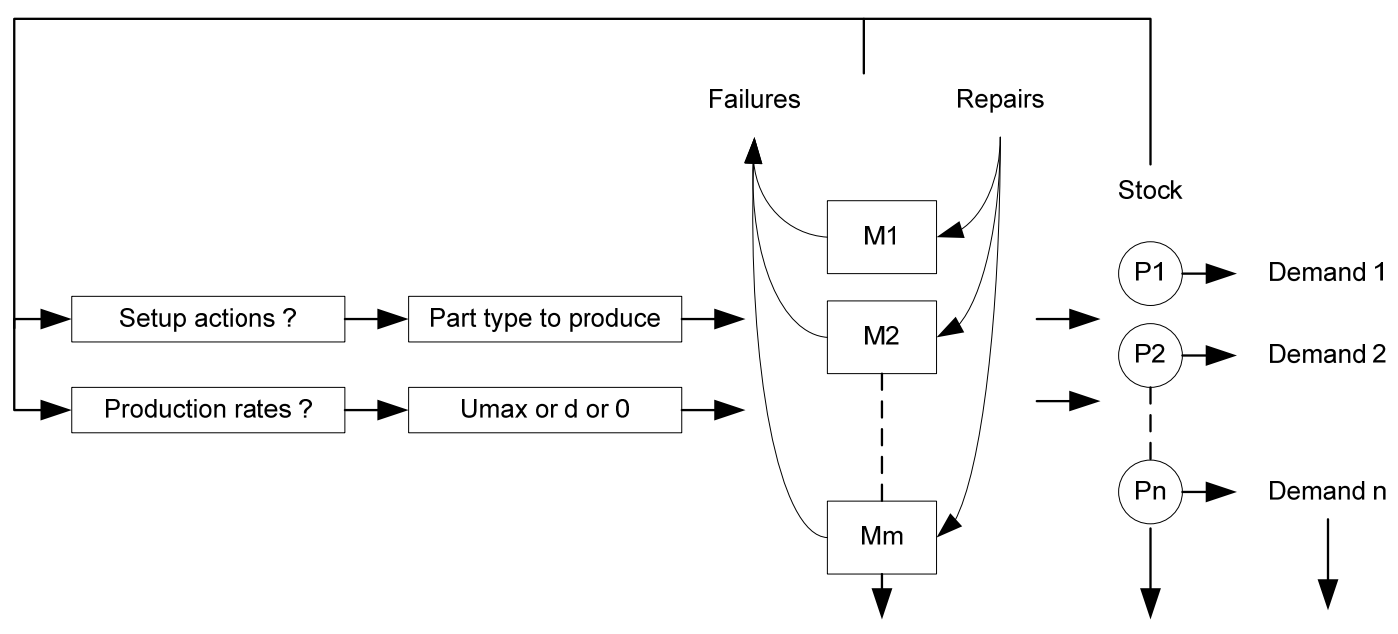

Total cost to minimize $=[$ Setup costs + inventory costs + backlog costs $]$

Figure 1: Structure of the manufacturing system under study 
Part type $i$ requires an average processing time $p_{i}>0,(i=1, \ldots, n)$ and has an average time between orders $1 / d_{i}$ assumed to be constant. For an $n$ part type system, $\boldsymbol{x}(\boldsymbol{t}), \boldsymbol{u}(\boldsymbol{t})$ and $\boldsymbol{d}$ denote vectors of the inventory/backlog levels $\left(x_{1}(t), \ldots, x_{n}(t)\right)^{\prime}$, production rates $\left(u_{1}(t), \ldots, u_{n}(t)\right)^{\prime}$ such that $u_{i}()=.\sum_{j=1}^{m} u_{i j}($.$) , and demand rates \left(d_{1}, \ldots, d_{n}\right)^{\prime}$ respectively.

The state of the system at time $t$ has two components, including a continuous part which describes the cumulative surplus vector, and is measured by $\boldsymbol{x}(t)$ and a discrete part, which describes machines states, and is denoted by $\alpha(\mathrm{t})=\left(\alpha_{1}(t), \ldots, \alpha_{m}(t)\right)^{\prime}$. The state of the stochastic process $\alpha_{j}(\mathrm{t})$ is equal to 0 if the machine $j$ is under repair and 1 if the machine is operational. For the manufacturing system considered, the state space is given by: $x(\mathrm{t}) \in \mathrm{R}^{n}, \alpha(\mathrm{t}) \in \mathrm{M}=M_{1} \times \ldots M_{m}$, with $M_{j}=\{0,1\}$.

The dynamic of the surplus is given by the following differential equation:

$$
\dot{x}(t)=u(t)-d, \quad x(0)=x
$$

where $x$ denotes the initial vector of surplus levels.

Machine $j$ uptimes and downtimes are assumed to be exponentially distributed with rates $p_{j}$ and $r_{j}$, respectively. The machine state evolves according to a continuous-time Markov process with modes in $M_{j}$ and with a generator matrix $Q$ such that: $Q=\left\{q_{\alpha \beta}\right\}$, where $q_{\alpha \beta}$ denotes the transition rates from modes $\alpha$ to $\beta$, with $q_{\alpha \beta} \geq 0$ if $\alpha \neq \beta$ and $q_{\alpha \alpha}=-\sum_{\beta \neq \alpha} q_{\alpha \beta}, \alpha, \beta \in M$. The transitions rates matrix $\boldsymbol{Q}$ is expressed as follows:

$$
Q=\left|\begin{array}{cc}
-q_{01} & q_{01} \\
q_{10} & -q_{10}
\end{array}\right| \text { with } q_{10}=p_{j} \text { and } q_{01}=r_{j} \text {. }
$$

The production rates at any given time must satisfy the capacity constraint of the system given by the following equation:

$$
0 \leq u_{i j}(t) \leq U_{i j}^{\max }(t), i=1 \ldots n, j=1 . . m
$$

Where $U_{i j}^{\max }(t)$ denotes the maximal production rate of product $\mathrm{i}$ on machine $\mathrm{j}$. 
For each $\alpha \in M$, the feasible production rates (or capacity) set is given by:

$$
\Gamma_{i}(\alpha)=\left\{u: u=\left(\mathrm{u}_{1}, \ldots, \mathrm{u}_{n}\right) \geq 0,0 \leq \mathrm{u}_{i j} \leq U_{i j}^{\max }(\mathrm{t}), \mathrm{u}_{k j}=0 ; \forall k \neq \mathrm{i}\right\}
$$

Our decision variables are production rates $\boldsymbol{u}()=.\left(u_{1}(),. \ldots, u_{n}().\right)$ and a sequence of setups denoted by $\Omega=\left\{\left(\tau_{0}, i_{0} i_{1}\right),\left(\tau_{1}, i_{1} i_{2}\right), \ldots\right\}$. A setup $(\tau, i j)$ is defined by the time $\tau$ at which it begins and a pair $i j$, denoting that the system was already set up to produce part $i$ and is being switched to be able to produce part $j$.

The instantaneous inventory and backlog cost function $g($.$) is given by the following$ equation:

$$
g(x)=\sum_{i=1}^{n}\left(c_{i}^{+} x_{i}^{+}+c_{i}^{-} x_{i}^{-}\right)
$$

where $x_{i}^{+}=\max \left(0, x_{i}\right), x_{i}^{-}=\max \left(-x_{i}, 0\right), c_{i}^{-}$: product type $i$ backlog cost and $c_{i}^{+}$: product type $i$ inventory cost.

Let $i$ denote the initial setup state of the system and $s$ the remaining setup time. The setup cost is assumed to be charged at the beginning of the setup.

The instantaneous setup cost during $s$ units of time is given by the following equation:

$$
R_{i j}(\mathrm{x}, s)=\sum_{k=1}^{m} K_{i j}^{k} I n d\left\{s=\Theta_{i j}^{k}\right\}+\int_{0}^{S} e^{-\rho t} g(\mathrm{x}-d t) d t, s \in\left[0, \Theta_{i j}^{k}\right], \mathrm{i}, \mathrm{j}=1, \ldots, \mathrm{n} \text { and } i \neq j
$$

where $\rho$ denotes the discounted rate of the incurred cost and Ind $\left\{s=\Theta_{i j}^{k}\right\}=\left\{\begin{array}{ll}1, & \text { if } \quad s=\Theta_{i j}^{k} \\ 0, & \text { otherwise }\end{array}\right.$. Using (3)-(4), the total cost $J($.$) can be defined by the following expression:$ 
$J(i, x, \alpha, s, \Omega, u())=.\int_{0}^{S} e^{-\rho t} g(x(t)) d t+E_{i, x-d s, \alpha_{s}}\left[\int_{S}^{\infty} e^{-\rho t} g(x(t)) d t+\sum_{k=1}^{m} \sum_{l=0}^{\infty} e^{-\rho \tau_{l}} K_{i_{l} i_{l+1}}^{k}\right](5)$

Let $A$ denote the set of admissible decisions $(\Omega, \boldsymbol{u}()$.$) . The production planning problem$ considered here is to find an admissible decision or control policy $(\Omega, \boldsymbol{u}()$.$) that$ minimizes $J($.$) , given by (5) subject to equations (1) to (3). Such a feedback control$ policy, as illustrated in Figure 1, determines the production rates and the setup actions as a function of the surplus level $\boldsymbol{x}$ and the state of the system $\alpha$.

While producing the part type $i$, the corresponding value function $v_{i}($.$) can be given by the$ following:

$$
v_{i}(x, \alpha, s)=\inf _{(\Omega, u) \in A} J(i, x, \alpha, s, \Omega, u) \quad \forall x \in R^{n}, \alpha \in M
$$

As in Sethi and Zhang (1994), it can be shown that the value function $v_{i}(x, \alpha)$ is locally Lipschitz, and is the unique viscosity solution to the following HJB equation:

$$
\min \left\{\begin{array}{l}
\min _{u \in \Gamma(\alpha)}\left[(u-d)\left(v_{i}\right)_{x}(x, \alpha)+g(x)+Q \cdot v_{i}(x, .)(\alpha)\right]-\rho v_{i}(x, \alpha) ; \\
\min _{j \neq i}\left[R_{i j}\left(x, \Theta_{i j}\right)+e^{-\rho \Theta_{i j}} \cdot v_{j}\left(x-d \Theta_{i j}, 1\right)\right]-v_{i}(x, \alpha)
\end{array}\right\}=0
$$

where $\left(v_{i}\right)_{x}($.$) denotes the gradients of v_{i}($.$) with respect to \boldsymbol{x}, \Theta_{i j}=\underset{k=1, \ldots, m}{\operatorname{Sup}}\left\{\Theta_{i j}^{k}\right\}$.

The production and setup policy that we are seeking is obtained when the value function is known. While we cannot analytically solve the HJB equations (7), we can however apply numerical methods to obtain the approximation of the value function and the associated control policy as in Yan and Zhang (1997).

\section{Numerical Approach and Optimal Control Policy}

In this section, numerical methods are used to approximate the solution of the HJB equations (7) corresponding to the stochastic optimal control problem, and to solve the corresponding optimality conditions. This method is based on the Kushner approach 
(Kushner and Dupuis (1992)). The basic idea behind it consists in using an approximation scheme for the gradient of the value function $v_{i}(x, \alpha)$.

Let $h_{j}, \mathrm{j}=1 \ldots \mathrm{n}$, denote the length of the finite difference interval of the variable $x_{j}$. Using the finite difference approximation, $v_{i}(x, \alpha)$ could be given by $v_{i}^{h}(x, \alpha)$, and the gradient $\left(v_{i}\right)_{x_{j}}(x, \alpha)$ by:

$$
\left(v_{i}\right)_{x_{j}}(x, \alpha)= \begin{cases}\frac{1}{h_{j}}\left(v_{i}^{h}\left(x_{1}, \ldots, x_{j}+h_{j}, . ., x_{n}\right)-v_{i}^{h}\left(x_{1}, . ., x_{j}, . ., x_{n}\right)\right) & \text { if } u_{j}-d_{j} \geq 0 \\ \frac{1}{h_{j}}\left(v_{i}^{h}\left(x_{1}, \ldots, x_{j}, \ldots, x_{n}\right)-v_{i}^{h}\left(x_{1}, \ldots, x_{j}-h_{j}, . ., x_{n}\right)\right) & \text { if } u_{j}-d_{j}<0\end{cases}
$$

We could see that:

$$
\begin{aligned}
\left(u_{j}-d_{j}\right)\left(v_{i}\right)_{X_{j}}(x, \alpha) & =\frac{\left|u_{j}-d_{j}\right|}{h_{j}} v_{i}^{h}\left(x_{1}, . ., x_{j}+h_{j}, . ., x_{n}\right) \operatorname{Ind}\left\{u_{j}-d_{j} \geq 0\right\} \\
& +\frac{\left|u_{j}-d_{j}\right|}{h_{j}} v_{i}^{h}\left(x_{1}, . ., x_{j}-h_{j}, . ., x_{n}\right) \operatorname{Ind}\left\{u_{j}-d_{j}<0\right\} \\
& -\frac{\left|u_{j}-d_{j}\right|}{h_{j}} v_{i}^{h}\left(x_{1}, . ., x_{j}, . ., x_{n}\right)
\end{aligned}
$$

With this approximation, the HJB equations (7) are expressed in terms of $v_{i}^{h}(x, \alpha)$, as shown in equation (8). Such an approximation is used in Hajji et al. (2004) to show that the HJB equations could be represented by the following equation:

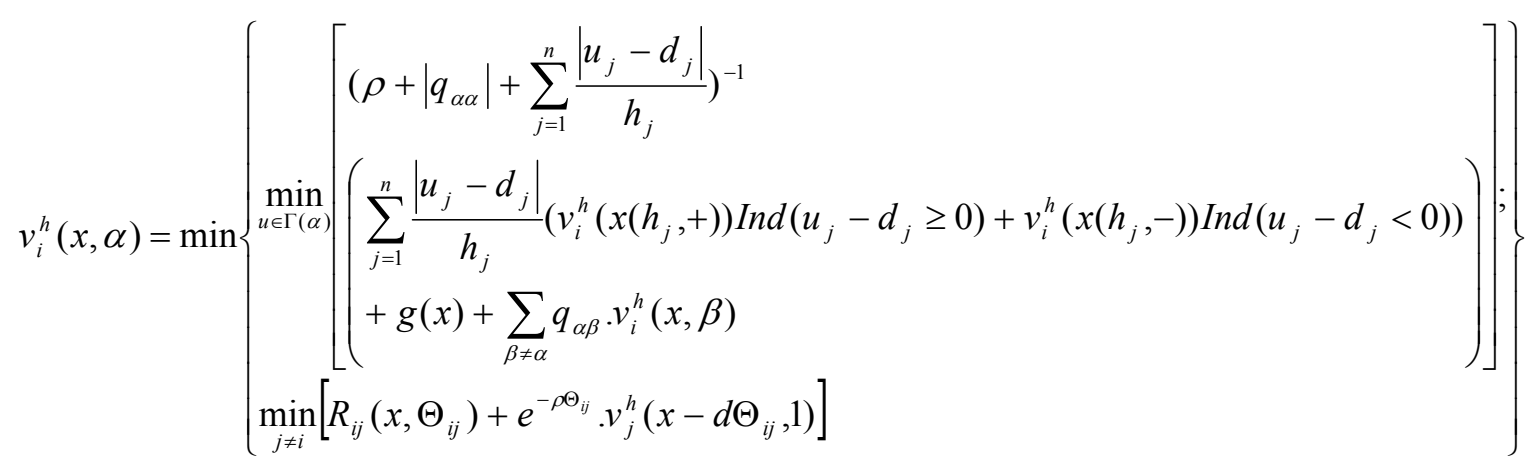

The solution of the numerical approximation of $v_{i}(x, \alpha)$ may be obtained by either successive approximation or policy improvement techniques (Boukas and Kenné (1997) and Kushner and Dupuis (1992)). 


\subsection{Complexity of the optimal control problem}

The dimension of the HJB equations for numerical methods is given by:

$$
\operatorname{Dim}=2^{m} \times 3^{m \times n} \times\left[\prod_{i=1}^{n} N_{h}\left(x_{i}\right)\right] \times 2^{n-1}
$$

Where $N_{h}\left(x_{i}\right)=\operatorname{card}\left[G_{h}\left(x_{i}\right)\right]$ with $G_{h}\left(x_{i}\right)$ describing the numerical grid for the state variable $x_{i}$ related to product $P_{i}, \mathrm{i}=1, \ldots$, n. Each machine has two states (i.e., $2^{m}$ states for a m-machine manufacturing system) and its production rate can take three values namely maximal production rate, demand rate and zero for each product (i.e., $3^{m \times n}$ states for a $m$ machine, $n$-product manufacturing system). While producing a product type, one of two possible decisions must be taken (i.e., $2^{n-1}$ for a $n$-product manufacturing system). Based on such dimension, the related numerical algorithm for the five-machine, two-product case is very difficult to implement and to solve. Such system is classified here as complex systems.

Due to the complexity of the HJB equations, the objective of this paper is not to solve them for the complex case, but to determine experimentally the parameters of a modified hedging corridor policy proved to be a best approximation of the optimal control policy than the classical one (i.e., Hedging corridor policy). It will be shown, in the next sections, and without loose of generality that the MHCP guarantee better performance than does the HCP for the one-machine two-product and the five-machine two-product manufacturing systems.

\subsection{Numerical results of the one-machine two-parts type case}

The implementation of the approximation technique requires the use of a finite grid denoted by $G_{h}$, where $h$ is a given vector of a finite difference interval. The considered computation domain $\mathrm{D}$ is given by:

$$
\mathrm{D}=\left\{\left(x_{1}, x_{2}\right):-5 \leq x_{1} \leq 5,-5 \leq x_{2} \leq 5, h_{1}=h_{2}=0.2\right\}
$$

To ensure a clear characterization of the control policy, several elements were taken into consideration as part of the implementation process. Indeed, the production and setup 
policies, in which the machine produces part type i for example, are each observed separately. For each policy, the relevant significant stock threshes are analyzed independently of the others. For each numerical result, the policies are provided as shown in Figure 2. The resulting production and setup policies (Figure 2 (a) and Figure 2 (b), respectively) divide the surplus space into the following three mutually exclusive regions:

- In region I, keep the same setup and produce the part type at the maximum or demand rate.

- In region II, change the configuration of the machine and produce the other part type.

- In region III, keep the same setup of the machine and set the production rate to zero.

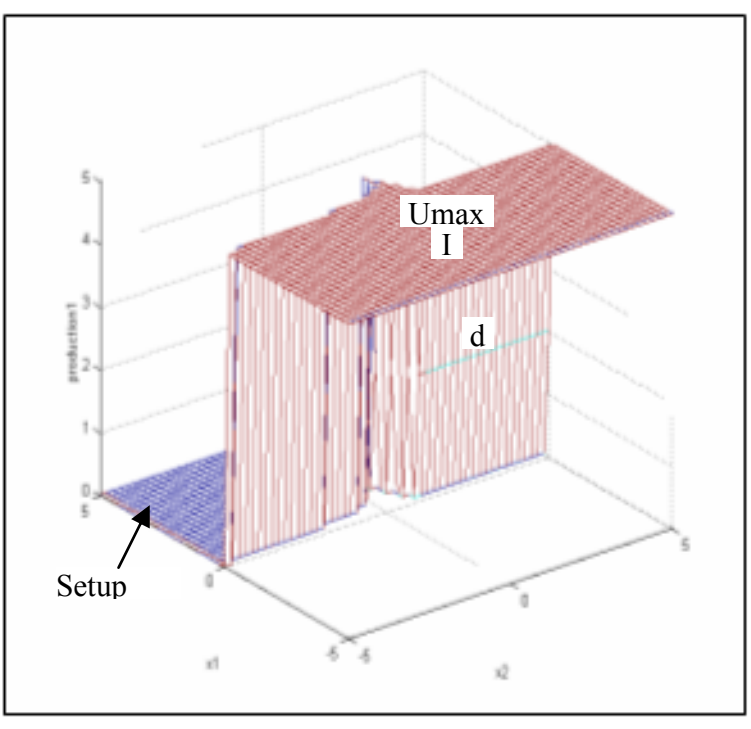

(a) Production policy

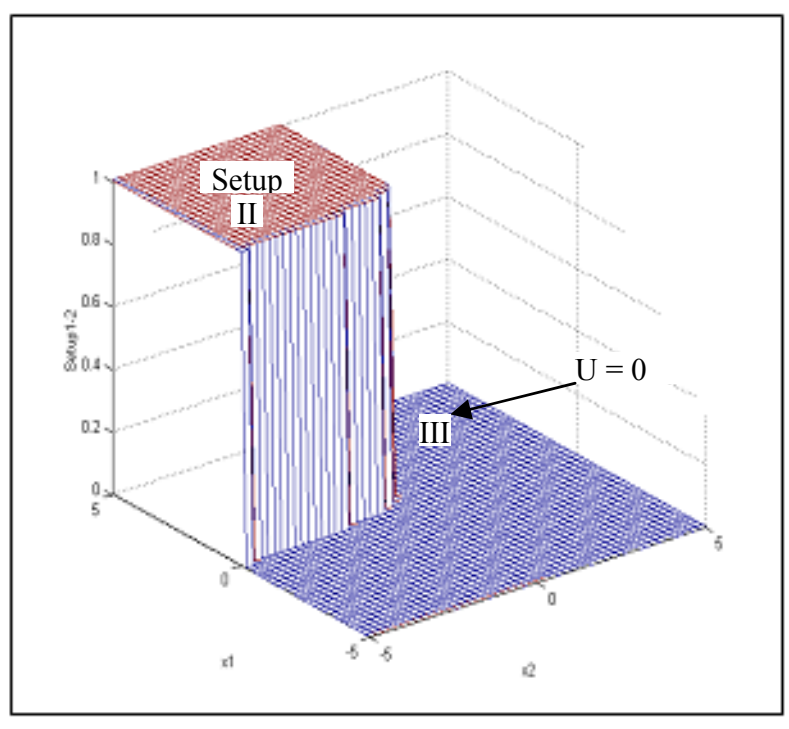

(b) Setup policy

Figure 2: Part type 1 production and setup policies

The numerical results used to characterize the optimal production and setup policies are analyzed in this section within several cases. Table 1 shows the constant parameters for all numerical examples considered and an illustrative case of variable parameters (i.e., inventory and backlog costs).

Table 1: Data parameters 


\begin{tabular}{|c|c|c|c|c|c|c|c|}
\hline Parameters & $\left(c_{i}^{+}, c_{i}^{-}\right)$ & $\left(K_{12}^{1}, K_{21}^{1}\right)$ & $\left(\Theta_{12}^{1}, \Theta_{21}^{1}\right)$ & $(p, r)$ & $\left(u_{11}^{\max }, u_{21}^{\max }\right)$ & $\left(d_{1}, d_{2}\right)$ & $\rho$ \\
\hline Values & $(1,5)$ & $(0.5,0.5)$ & $(0.16,0.16)$ & $(0.15,0.8)$ & $(5,5)$ & $(2,2)$ & 0.9 \\
\hline
\end{tabular}

It follows from our numerical results that the optimal policy has a particular structure, which we call here the Modified Hedging Corridor Policy "MHCP" and illustrated in Figure 3. This policy is a combination of the Hedging Corridor Policy (HCP) and the Hedging Point Policy (HPP). Let Z1 and Z2 define the threshold of products P1 and P2 respectively. Let also and a1 and a2 define the boundary of the corridor.

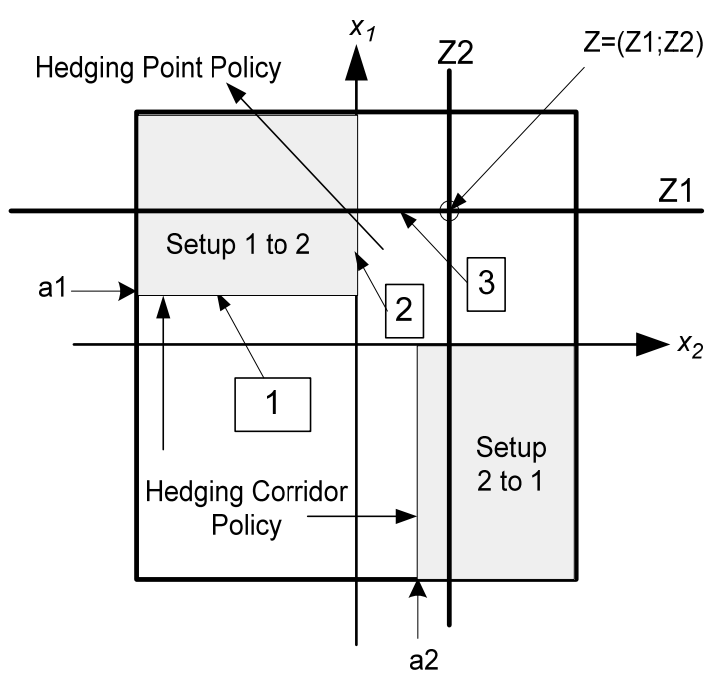

(a) Setup actions through MHCP

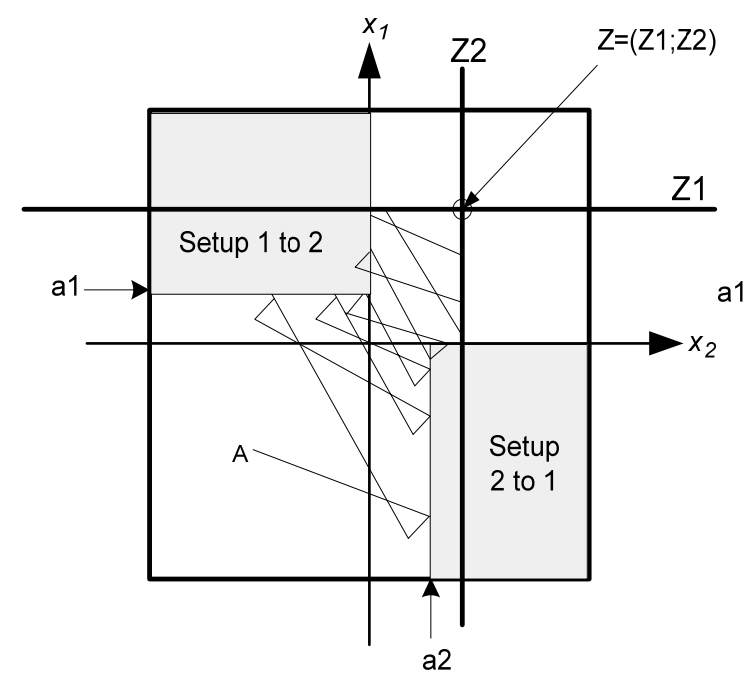

(b) Inventory itinerary through MHCP

Figure 3: Modified Hedging Corridor policy

The results obtained are different from those found by Yan and Zhang (1997) and Bai and Elhafsi (1997). The proposed MHCP is defined by two hedging levels in the negative and positive areas of each part type. When there is a shortage in the stock level of one part type, the setup action must be performed earlier than the same action when the stock level is still in the positive zone. These actions are conducted with respect to the boundaries of the corridors $a_{1}$ and $a_{2}$ (see arrow 1, Figure 3 (a)). However, when the two stock levels are positive, we can proceed with production according to a hedging point policy (see arrow 3, Figure 3 (a)), in this case the setup actions are performed when the stock level of the concerned part type reaches the negative area (see arrow 2, Figure 3 
(a)). The proposed modified hedging corridor policy, as shown in Figure 3 (b), states that if starting at point $\mathrm{A}$ and reaching point $\mathrm{Z}$, which is the hedging point defined by the intersection of the two hedging levels, the stock trajectory travels through two different corridors, with one in the negative and combined stock area and the other in the positive area.

\subsection{Sensitivity analysis: Parameterized control policy}

To illustrate the effect that changing the cost parameters has on the policy observed, a sensitivity analysis (Table 2) has been performed. It shows that when backlog costs rise, the value of the hedging thresholds and the boundary levels increase accordingly to ensure the availability of enough stocks to hedge against future backlogs. This observation is confirmed by the numerical threshold levels $Z_{1}^{*}, Z_{2}^{*}$ and setup boundaries $a_{1}^{*}, a_{2}^{*}$ presented in Table 2 (set I, basic case and cases 1 to 3 ). Moreover, when the inventory costs increase, the values of the hedging thresholds and the boundary levels decrease to confine the stock accumulation. This observation is confirmed by the numerical threshold levels $Z_{1}^{*}, Z_{2}^{*}$ and setup boundaries $a_{1}^{*}, a_{2}^{*}$ presented in Table 2 (set II, basic case and cases 1 to 3 ).

Table 2: Data parameters for the sensitivity analysis cases

\begin{tabular}{|c|c|c|c|c|c|c|c|}
\hline & Cases & $c_{1}^{+}$ & $c_{1}^{-}$ & $c_{2}^{+}$ & $c_{2}^{-}$ & $\left(Z_{1}^{*}, Z_{2}^{*}\right)$ & $\left(a_{1}^{*}, a_{2}^{*}\right)$ \\
\hline \multirow{4}{*}{ Set I } & Basic & 1 & 5 & 1 & 5 & $(1.8,1.8)$ & $(0.2,0.2)$ \\
\cline { 2 - 8 } & 1 & 1 & 10 & 1 & 10 & $(2,2)$ & $(0.3,0.3)$ \\
\cline { 2 - 8 } & 2 & 1 & 30 & 1 & 30 & $(2.2,2.2)$ & $(0.4,0.4)$ \\
\cline { 2 - 8 } & 3 & 1 & 60 & 1 & 60 & $(2.6,2.6)$ & $(0.5,0.5)$ \\
\hline \multirow{4}{*}{ Set II } & Basic & 1 & 60 & 1 & 60 & $(2.6,2.6)$ & $(0.5,0.5)$ \\
\cline { 2 - 8 } & 1 & 5 & 60 & 5 & 60 & $(1.8,1.8)$ & $(0.4,0.4)$ \\
\cline { 2 - 8 } & 2 & 10 & 60 & 10 & 60 & $(1.2,1.2)$ & $(0.3,0.3)$ \\
\cline { 2 - 8 } & 3 & 20 & 60 & 20 & 60 & $(0.6,0.6)$ & $(0.2,0.2)$ \\
\hline
\end{tabular}

From the above analysis, it clearly appears that the results obtained make sense, and that the structure of the policy defined by the 4 parameters $\left(a_{1}, a_{2}, Z_{1}\right.$ and $\left.Z_{2}\right)$ is always maintained. This allows the development of a parameterized production and setup control policy defined by the following equations: 


$$
\begin{aligned}
& u_{1}(.)= \begin{cases}u_{1}^{\max } \cdot \operatorname{Ind}\left\{S_{21}=1\right\} & \text { if } x_{1}<Z_{1} \\
d_{1} \cdot \operatorname{Ind}\left\{S_{21}=1\right\} & \text { if } x_{1}=Z_{1} \\
0 & \text { if } x_{1}>Z_{1}\end{cases} \\
& u_{2}(.)=\left\{\begin{array}{lc}
u_{2}^{\max } \cdot \operatorname{Ind}\left\{S_{12}=1\right\} & \text { if } x_{2}<Z_{2} \\
d_{2} \cdot \operatorname{Ind}\left\{S_{12}=1\right\} & \text { if } x_{1}=Z_{2} \\
0 & \text { if } x_{2}>Z_{2}
\end{array}\right. \\
& S_{12}=\left\{\begin{array}{l}
1 \quad \text { if }\left\{\begin{array}{l}
x_{1} \geq a_{1} \\
\text { and } \\
x_{2} \leq 0
\end{array}\right. \\
0 \quad \text { otherwise }
\end{array}\right. \\
& S_{21}=\left\{\begin{array}{l}
1 \quad \text { if }\left\{\begin{array}{l}
x_{2} \geq a_{2} \\
\text { and } \\
x_{1} \leq 0
\end{array}\right. \\
0 \quad \text { otherwise }
\end{array}\right.
\end{aligned}
$$

with the following constraints:

$$
0 \leq a_{1} \leq Z_{1} \text { and } 0 \leq a_{2} \leq Z_{2}
$$

The modified hedging corridor policy presented by equations (9) to (12) is completely defined for given values of $a_{i}$ and $Z_{i}(\mathrm{i}=1,2)$, called here design factors. The next sections are aimed at developing a systematic approach for determining optimal values of $a_{i}$ and $Z_{i}(\mathrm{i}=1,2)$.

\section{Control Approach}

In order to bring an approach which could be easily applied to control manufacturing systems at the operational level, the descriptive capacities of discrete event simulation models are combined with analytical models, experimental design, and response surface methodology. A block diagram of the resulting control approach is depicted in Figure 4. This approach has been successfully used to control production and perform preventive maintenance activities in the cases of single-machine and multiple-identical-machine flexible manufacturing systems (see Kenne and Gharbi (1999) and Gharbi and Kenne (2000)). 


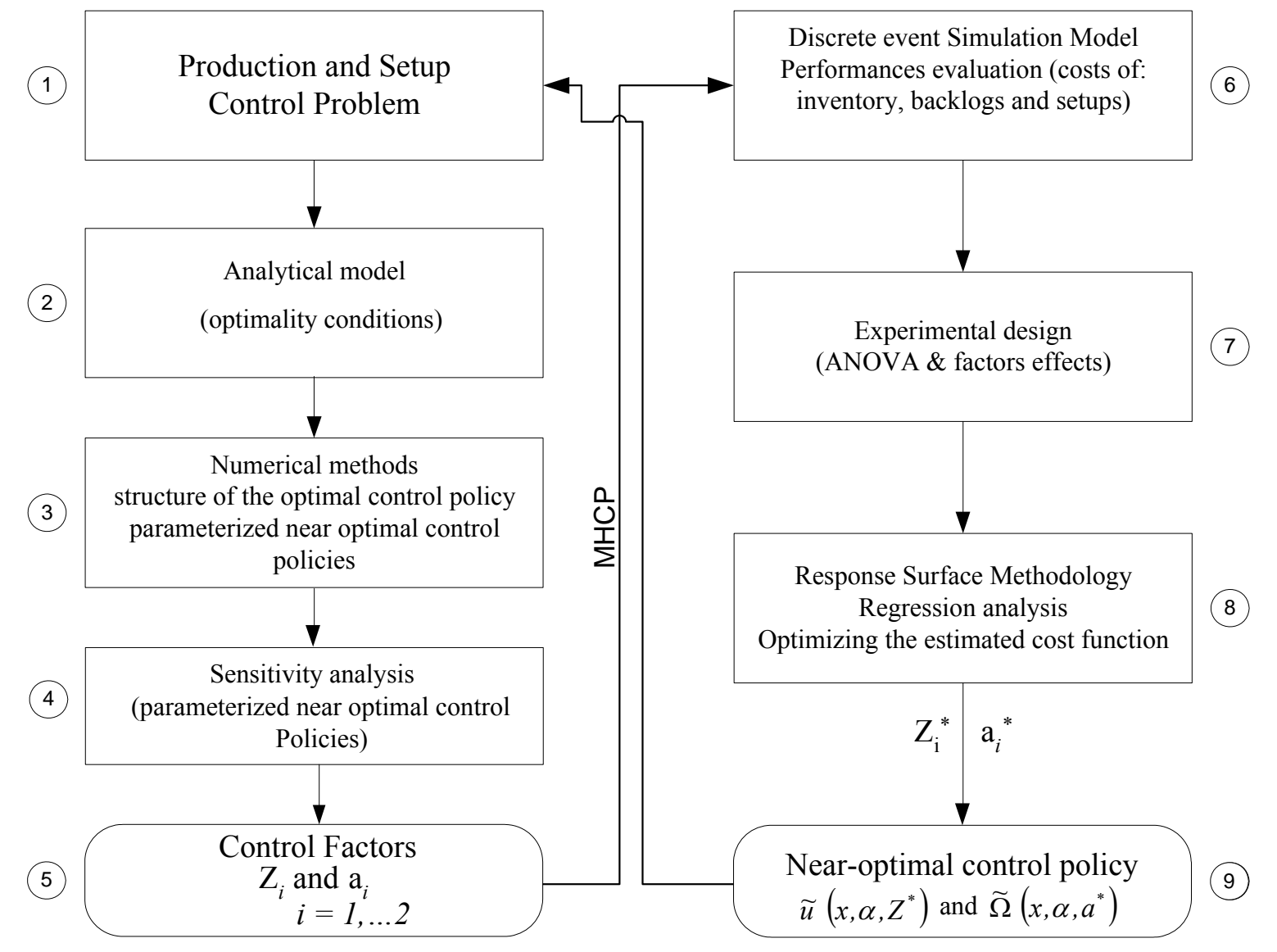

Figure 4: Proposed control approach

The structure of the proposed control approach presented in Figure 4 consists of the following sequential steps:

1. The Control problem statement of the manufacturing system, as shown in section 2 , consists of a representation of the production and setup control problem through a stochastic optimal control model based on the control theory. Hence, the problem of the optimal flow control for the manufacturing system considered is described in this first step, which contains a specification of the objective of the study. That objective is to find the control variables $(\boldsymbol{u}(\boldsymbol{x}, \boldsymbol{\alpha}), \Omega(\boldsymbol{x}, \boldsymbol{\alpha}))$, called the production rates and setup actions, in order to improve the related output (i.e., the incurred cost).

2. The optimality conditions, described by the HJB equations, are obtained from the problem statement of the previous step. It is shown in this step that the value function, 
representing the incurred cost, is the solution of the HJB equations, and the corresponding control policy (production rates and setup actions) is optimal.

3. The numerical methods are used in this step to solve the optimality equations of the problem, given that there is no way of solving them analytically.

4. The sensitivity analysis is conducted to illustrate the effects that changing certain parameters has on the numerical results. It ensures the proper characterization of the control policy structure so as to develop a parameterized policy.

5. The control factors $\mathrm{Z}_{i}, i=1, \ldots 2$ for production rates control and $\mathrm{a}_{i}, i=1, \ldots 2$ for setup actions control, describe the numerical control policy obtained.

6. The simulation model uses the near-optimal control policy defined in the previous step as the input for conducting experiments in order to evaluate the performances of the manufacturing system. Hence, for given values of the control factors, the cost incurred is obtained from the simulation model presented later in section 5.

7. The experimental design approach defines how the control factors can be varied in order to determine the effects of the main factors and their interactions (i.e., analysis of variance or ANOVA) on the cost through a minimal set of simulation experiments.

8. The response surface methodology is then used to obtain the relationship between the incurred cost and significant main factors and interactions given in the previous step. The obtained model is then optimized in order to determine the best values of factors called here $\mathrm{Z}_{i}{ }^{*}$ for production, and $\mathrm{a}_{i}{ }^{*}$ for setup actions.

9. The near-optimal control policy $\left(\widetilde{u}\left(x, \alpha, Z^{*}\right), \widetilde{\Omega}\left(x, \alpha, a^{*}\right)\right)$ is thus an improved Modified Hedging Corridor Policy to be applied to the manufacturing system.

The application of the proposed control approach gives the production rates and setup actions described by equations (9), (10) and (11), (12) respectively, for the best values of factors $Z_{i}$ and $a_{i}$ (i.e., $Z_{i}^{*}$ and $a_{i}{ }^{*}$ ).

\section{Simulation Model}

A discrete event simulation model which describes the continuous dynamics of the system (1) and its discrete stochastic behavior, is developed using the Visual SLAM language (Pritsker and O'Reilly (1999)). This model consists of several networks, each of which describes a specific task in the system (i.e., demand generation, control policy, 
states of the machines, inventory control..., etc.). The diagram of the proposed simulation model is shown in Figure 5 with the following block notation descriptions:

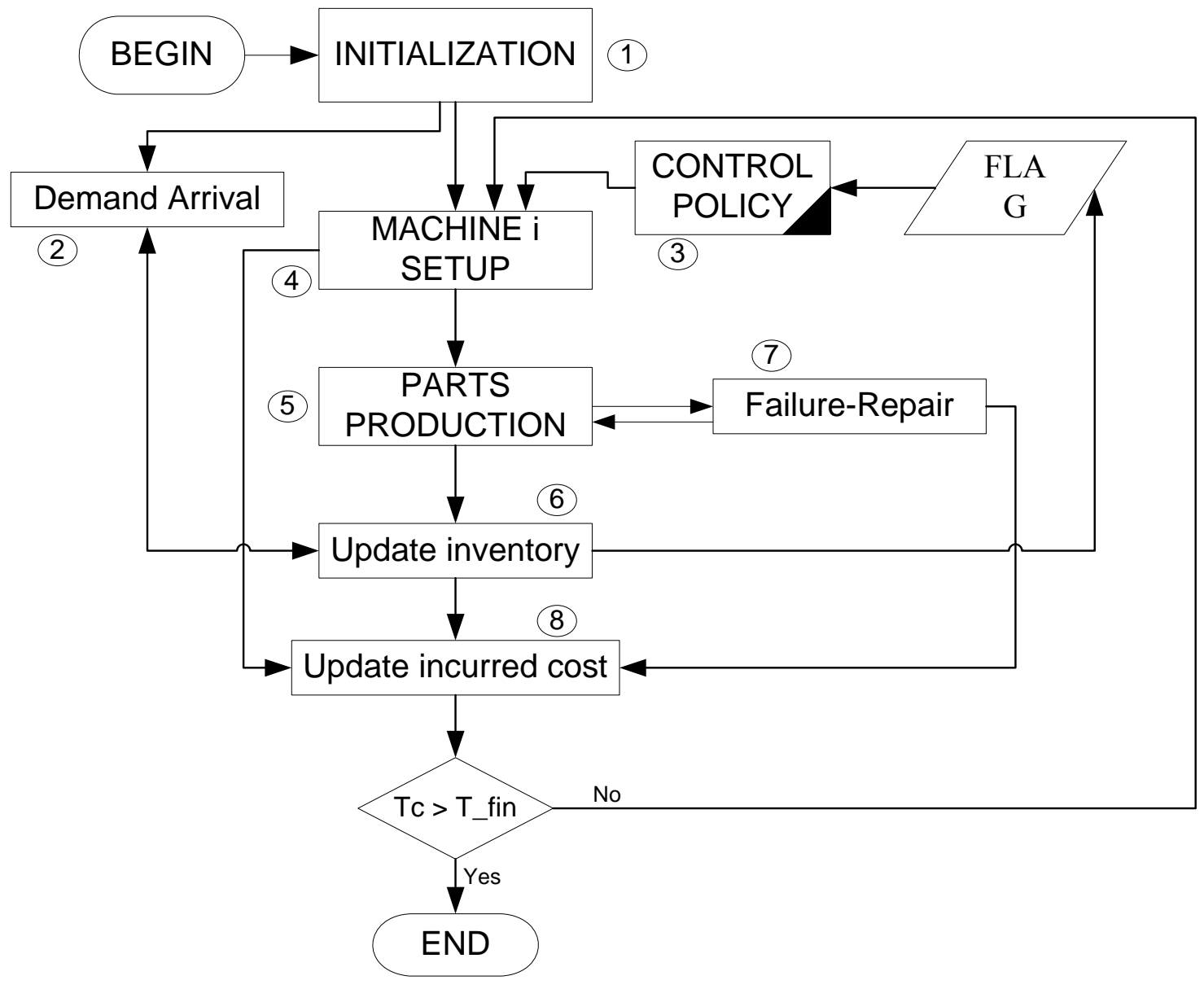

Figure 5: Diagram of simulation model

1. The INITIALIZATION block initializes the problem variables (current surplus, production rates, incurred cost, product type to start with...etc.)

2. The Demand Arrival block performs the arrival of a demand for product $j$ at each $\mathrm{d}_{\mathrm{j}}^{-1}$ unit of time. A verification test is then performed on the inventory level of product $j$, and the inventory or the backorder is updated.

3. The CONTROL POLICY segment block is defined in section 3 (see equations (9) to (12) for the machine production rates and setup actions). The feedback control policy is defined by the output of the FLAG block. This block is used to permanently verify 
the variation in the stock level $x_{j}(t)$ in order to specify the best action to carry out (production rate and setup actions).

4. The MACHINE SETUP block performs the setup of the machine $\mathrm{i}$ according to the policy defined by the CONTROL POLICY block.

5. The PARTS PRODUCTION block performs the production of finished goods according to the policy defined by the CONTROL POLICY block.

6. The update inventory block performs the variation of the inventory level when a part is produced or when a demand arrival occurs (i.e., production of finished goods increases inventory if there is no backorder or it satisfies the cumulative demands, and hence decreases backorders). Off-line runs of the simulation model, for a onemachine, two-parts type manufacturing system, using the control policy described by (9) to (12) for $Z_{i}=10$ and $\mathrm{a}_{i}=5, i=1,2$, is illustrated in Figure 6. We should recall that production and setup actions are conducted with respect to the hedging levels $Z_{1}$ and $Z_{2}$ and the boundaries of the corridor $a_{1}$ and $a_{2}$. The setup actions are performed according to a hedging corridor policy in the negative and the combined zones of the inventory (arrow 1, Figure 6). When the two stock levels are positive, we can produce according to a hedging level policy (arrow 3, Figure 6), in this case the setup actions are performed when the stock level of the concerned part type reaches the negative area (arrow 2, Figure 6). It is interesting to note that arrows 1 to 3 in Figure 6 represent the same phenomena observed in Figure 3 (a) and pointed out by arrows 1 to 3 . 


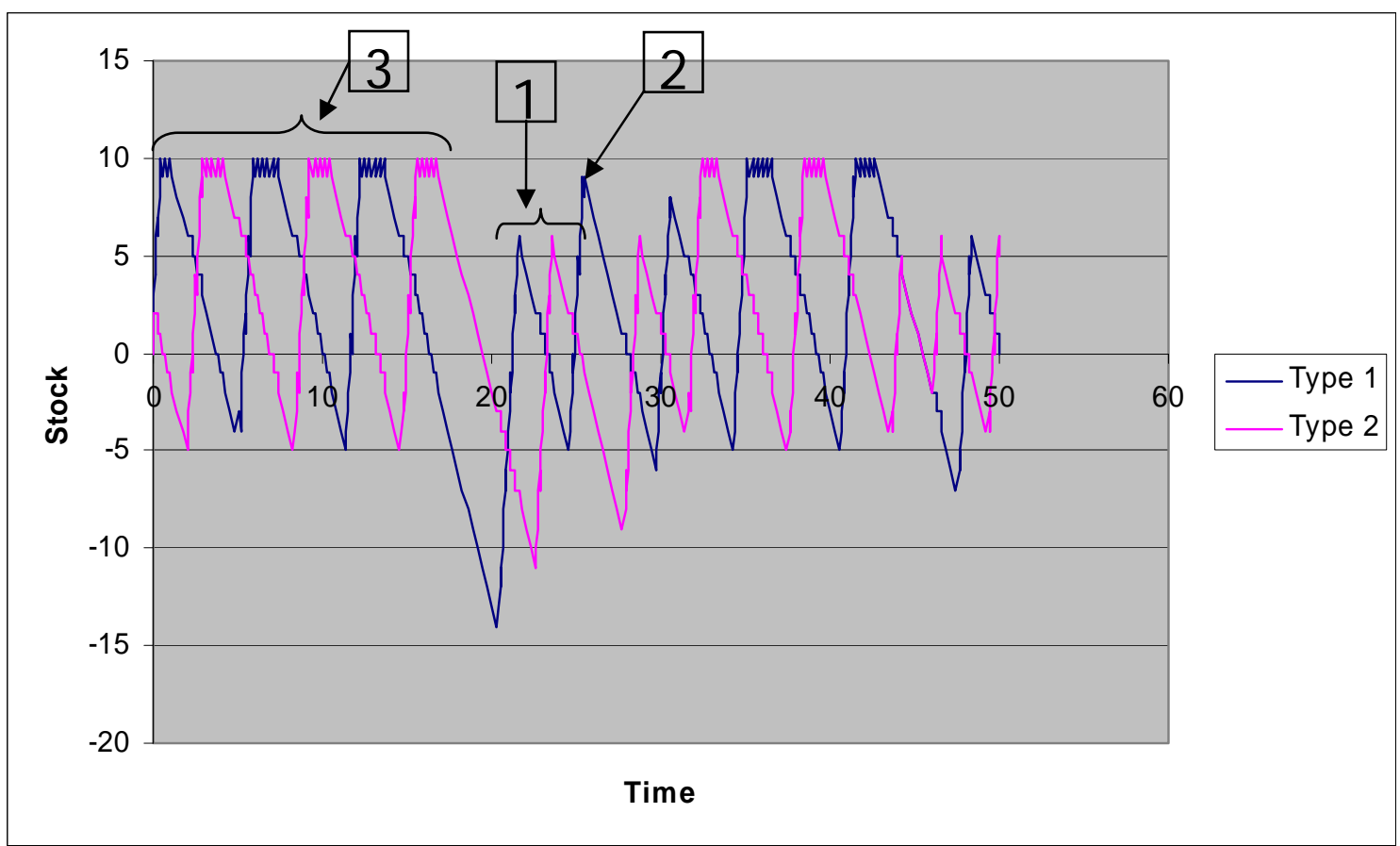

Figure 6: Two-product stock trajectory using $\mathrm{MHCP}(\mathrm{Z1}=\mathrm{Z2}=10$ and $\mathrm{a1}=\mathrm{a} 2=5)$

7. The failure-repair block performs two functions: it defines the time-to-failure of the machine, and repairs a broken one.

8. The update the incurred cost block calculates the cost of inventory, backlogs and setup actions.

In the next section, we present the procedure for varying the control factors simultaneously so as to obtain the appropriate relationship between the incurred cost and significant main factors and interactions. Such a procedure is known as the experimental design and response surface methodology approach.

\section{Experimental Design and Response Surface Methodology}

The objectives of this section are to: (i) determine whether the input parameters affect the response, (ii) estimate the relationship between the cost and significant factors, and finally, (iii) compute the optimal values of estimated factors. 
For the identical product type case (i.e., $c_{1}^{+}=c_{2}^{+}, c_{1}^{-}=c_{2}^{-}, K_{12}=K_{21}, \Theta_{12}=\Theta_{21}$, $u_{1}^{\max }=u_{2}^{\max }$ and $d_{1}=d_{2}$ ), we obtained $Z_{1}=Z_{2}$ and $a_{1}=a_{2}$. In this particular case, the control policy is defined by two design factors (a and Z) instead of four.

\subsection{Experimental design}

In this study, we collect and analyze data for a steady state cost which as much as possible approximates that defined by the value function given by equation (6). $\alpha=\frac{a}{Z}$ (with $\left.0 \leq \alpha \leq 1\right)$ is an independent variable defined such as to ensure that the constraints (13) are respected. We could see that:

$$
\left.\begin{array}{c}
\alpha=\frac{a}{Z} \\
\text { and } \\
0 \leq \alpha \leq 1
\end{array}\right\} \Rightarrow 0 \leq a \leq Z
$$

The experimental design is concerned with (i) selecting a set of input variables (i.e., factors $\alpha$ and Z) for the simulation model; (ii) setting the levels of selected factors of the model and making decisions on the conditions, such as the length of runs and number of replications, under which the model will be run.

Two independent variables and one dependent variable (the cost) are considered. The levels of independent variables or design factors must be carefully selected to ensure they properly represent the domain of interest. Due to the convexity property of the value function (6) (Sethi and Zhang (1994)), the first-order response surface model is rejected. Hence, we selected a $3^{2}$-response surface design since we have 2 independent variables, each at three levels. The levels of the independent variables were selected as in Table 3.

Table 3: Levels of the independent variables

\begin{tabular}{|c|c|c|c|}
\hline & Low level & Center & High level \\
\hline$\alpha=\mathrm{a} / \mathrm{Z}$ & 0.1 & 0.5 & 0.9 \\
\hline $\mathrm{Z}$ & 6 & 18 & 30 \\
\hline
\end{tabular}


Four replications were conducted for each combination of the factors, and therefore, 36 $\left(3^{2} \times 4\right)$ simulation runs were made. To reduce the number of replications, we used a variance reduction technique called common random numbers (Law and Kelton (2000)). We conducted some preliminary simulation experiments using 4 replications, and noticed that the variability allows the effects to be distinguished. It is interesting to note that all possible combinations of different levels of factors are provided by the response surface design considered. The experimental design is used to study the effects that some parameters, namely $\alpha$ and Z, and their interactions have on the performance measure (i.e., the cost).

\subsection{Statistical analysis}

The statistical analysis of the simulation data consists of the multi-factor analysis of the variance (ANOVA). This is done using a statistical software application such as STATGRAPHICS, to provide the effects of the two independent variables on the dependent variable. Table 4 illustrates the ANOVA for $c_{1}^{+}=c_{2}^{+}=5$ and $c_{1}^{-}=c_{2}^{-}=15$.

From Table 4, we can see that the main factors $\alpha$ and Z, their quadratic effects, as well as their interactions are significant at the 0.05 level (i.e., P-value $<0.05$; symbol $\mathrm{S}$ in the last column). One more result that stands out in the ANOVA table is the blocks effect, which appears to be non-significant (symbol NS in the last column). This effect is due to the aforementioned variance reduction technique. The technique guarantees the generation of the same sequence of random numbers, thus the same failure and repair times, within the different runs of one block (one replication). However, a different sequence of random numbers is generated from one block to another (one replication to another). Consequently, it was expected that the block effect would be non-significant.

The residual analysis was used to verify the adequacy of the model. A residual versus predicted value plot and normal probability plot were used to test the homogeneity of the variances and the residual normality, respectively. We concluded that the normality and equality of variance could be improved. Thus, a data transformation was conducted. An analysis of the square of the response variable led to satisfactory plots. Moreover, the Rsquared value increased from 0.91 before transformation to 0.951 after transformation, as 
presented in Table 4. This indicates that more than $95 \%$ of the total variability is explained by the model (Montgomery (2001)), which is very satisfactory. The model obtained includes two main factors ( $\alpha$ and Z), two quadratic effects ( $\alpha^{2}$ and $Z^{2}$ ), and the interaction effect $\alpha \times Z$.

Table 4: ANOVA table

\begin{tabular}{|c|c|c|c|c|c|c|}
\hline Source & Sum of Squares & Df & Mean Square & F-Ratio & P-Value & \\
\hline 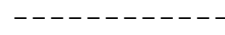 & - - - - - - - - - - - & -- & - - - - - - - - - & ---- & 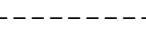 & -- \\
\hline A:Alpha & $1,47224 \mathrm{E} 10$ & 1 & 1,47224E10 & 335,61 & $\odot, \odot \odot \odot \odot$ & $\mathrm{S}$ \\
\hline$B: Z$ & $4,74927 \mathrm{E} 9$ & 1 & $4,74927 \mathrm{E} 9$ & 108,26 & 0,0000 & $\mathrm{~S}$ \\
\hline $\mathrm{AA}$ & $2,2597 \mathrm{E} 9$ & 1 & $2,2597 \mathrm{E} 9$ & 51,51 & 0,0000 & $\mathrm{~S}$ \\
\hline$A B$ & $2,08089 \mathrm{E} 8$ & 1 & $2,08089 E 8$ & 4,74 & 0,0383 & $\mathrm{~S}$ \\
\hline $\mathrm{BB}$ & $1,34277 \mathrm{E} 9$ & 1 & $1,34277 \mathrm{E} 9$ & 30,61 & 0,0000 & $\mathrm{~S}$ \\
\hline blocks & $2,24566 \mathrm{E} 7$ & 3 & $7,48554 \mathrm{E} 6$ & 0,17 & $\odot, 9153$ & NS \\
\hline Total error & $1,18444 \mathrm{E} 9$ & 27 & $4,38682 \mathrm{E} 7$ & & & \\
\hline
\end{tabular}

\subsection{Response surface methodology}

The Response surface methodology is a collection of mathematical and statistical techniques that are useful for modeling and analyzing problems in which a response of interest is influenced by several variables, and the objective is to optimize this response (Montgomery (2001)). We assume here that there exists a function $\Phi$ of $\alpha$ and $\mathrm{Z}$ that provides the value of the cost corresponding to any given combination of input factors, i.e., $\operatorname{Cost}^{2}=\Phi(\alpha, Z)$.

The function $\Phi($.$) is called the response surface, and is assumed to be a continuous$ function of $\alpha$ and $\mathrm{Z}$. The second order model is thus given by:

$$
\Phi=\beta_{0}+\beta_{11} \alpha+\beta_{12} Z+\beta_{21} \alpha^{2}+\beta_{22} Z^{2}+\beta_{3} \alpha \cdot Z+\varepsilon
$$

where $\alpha$ and $\mathrm{Z}$ are the input variables; $\beta_{0}, \beta_{11}, \beta_{12}, \beta_{21}, \beta_{22}$ and $\beta_{3}$ are unknown parameters, and $\varepsilon$ is a random error. From STATGRAPHICS, the estimation of unknown parameters is performed, and the following six coefficients achieved. The values of these coefficients are: 
$\beta_{0}=138448.0, \quad \beta_{11}=-180484, \quad \beta_{1}^{2}=-4786.81, \quad \beta_{21}=105041.0, \quad \beta_{22}=89.96, \quad$ and $\beta_{3}=751.31$. The corresponding response surface is presented in Figure 7. The optimum is obtained for $\alpha^{*}=0.77$ and $Z^{*}=23$, and the incurred square cost $\Phi^{*}$ is 12540.3 . Thus, $a^{*}=17$ and $Z^{*}=23$, and the incurred cost is $\sqrt{\Phi^{*}}=112$.

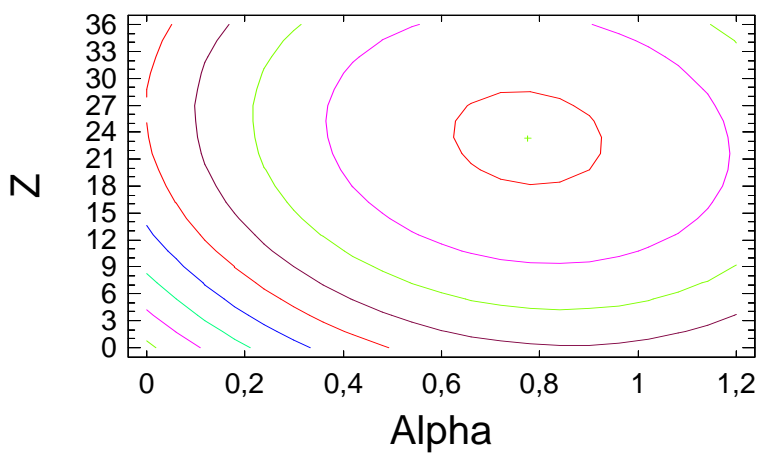

Figure 7: Cost response surface

\subsection{Sensitivity analysis}

To illustrate the effect of the cost variation on the design parameters, a sensitivity analysis was conducted. Table 5 details the cost variations, and presents the optimal parameters and the incurred optimal costs for the sensitivity analysis cases. It clearly appears that the results obtained make sense and confirm the numerical observation in the sense that when the backlog cost increases, cases 3 to 5, (resp. decreases, cases 3 to 1), the hedging levels and the corridor boundaries increase (resp. decrease).

Table 5: Optimal design factors and incurred costs for the sensitivity analysis cases with MHCP

\begin{tabular}{|c|c|c|c|c|c|c|c|c|c|}
\hline Cases & $K_{i j}$ & $c_{1}^{+}$ & $c_{1}^{-}$ & $c_{2}^{+}$ & $c_{2}^{-}$ & $\mathrm{a}^{*}=\alpha . \mathrm{Z}$ & $\mathrm{Z}^{*}$ & $c_{M H C P}^{*} \S$ & Remark \\
\hline 1 & 30 & 5 & 8 & 5 & 8 & 11 & 15 & 83 & $Z^{*} \downarrow, a^{*} \downarrow$ \\
\hline 2 & 30 & 5 & 10 & 5 & 10 & 13 & 18 & 90 & $Z^{*} \downarrow, a^{*} \downarrow$ \\
\hline 3 & 30 & 5 & 15 & 5 & 15 & 17 & 23 & 112 & Basic case \\
\hline 4 & 30 & 5 & 20 & 5 & 20 & 20 & 25 & 119 & $Z^{*} \uparrow, a^{*} \uparrow$ \\
\hline 5 & 30 & 5 & 25 & 5 & 25 & 21 & 26 & 125 & $Z^{*} \uparrow, a^{*} \uparrow$ \\
\hline
\end{tabular}

$\S c_{M H C P}^{*}$ the optimal incurred cost under MHCP

In the next section, a comparison between the MHCP and HCP is conducted. 


\subsection{Comparison of MHCP and HCP}

The hedging corridor policy (Bai and Elhafsi (1997)) is presented in Figure 8. The structure of such a policy is defined by two thresholds related to the two-part type. This corridor guides the surplus trajectory to target positive stock thresholds built up to hedge against future capacity shortages brought about by machine failures and setups.

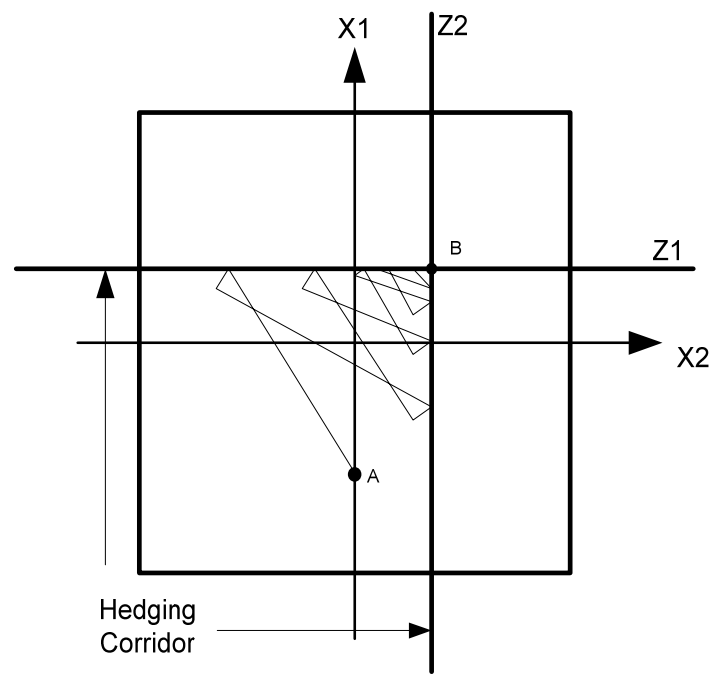

Figure 8: Hedging Corridor Policy

We present in Table 6 the incurred optimal costs for the same sensitivity analysis input, conducted with the Hedging Corridor Policy (HCP). It is important to note that the results presented in Table 6 were obtained under the same conditions (simulation, experimental design and RSM), and following the same approach under which the sensitivity analysis was conducted for the MHCP (table 5).

Table 6: Optimal design factors and incurred costs for the sensitivity analysis cases with $\mathrm{HCP}$

\begin{tabular}{|c|c|c|c|c|c|c|c|c|}
\hline Cases & $K_{i j}$ & $c_{1}^{+}$ & $c_{1}^{-}$ & $c_{2}^{+}$ & $c_{2}^{-}$ & $Z^{*}$ & $c_{H C P}^{*} \S$ & Remark \\
\hline 1 & 30 & 5 & 8 & 5 & 8 & 14 & 89.4 & $Z^{*} \downarrow, c_{H C P}^{*}>c_{M H C P}^{*}$ \\
\hline 2 & 30 & 5 & 10 & 5 & 10 & 16 & 98.6 & $Z^{*} \downarrow, c_{H C P}^{*}>c_{M H C P}^{*}$ \\
\hline 3 & 30 & 5 & 15 & 5 & 15 & 18 & 114 & Basic case, $c_{H C P}^{*}>c_{M H C P}^{*}$ \\
\hline 4 & 30 & 5 & 20 & 5 & 20 & 20 & 123.4 & $Z^{*} \uparrow, c_{H C P}^{*}>c_{M H C P}^{*}$ \\
\hline 5 & 30 & 5 & 25 & 5 & 25 & 21 & 130 & $Z^{*} \uparrow, c_{H C P}^{*}>c_{M H C P}^{*}$ \\
\hline
\end{tabular}

$\S c_{H C P}^{*}$ the optimal incurred cost under HCP 
The results obtained show that under HCP, the variation of the design parameter does make sense. Thus, when the backlog cost increases, cases 3 to 5, (resp. decreases, cases 3 to 1), the hedging levels increase (resp. decrease). However, the incurred costs for all the cases are higher than those incurred under MHCP. To confirm the numerical observation and hence the advantage of the proposed MHCP policy compared to that of HCP, a student test was performed in order to compare the performance of the two policies. The confidence interval of $c_{H C P}^{*}-c_{M H C P}^{*}$ is given by (15).

$$
\begin{gathered}
\bar{C}_{H C P}^{*}-\bar{C}_{M H C P}^{*}-t_{\alpha / 2, n-1} \operatorname{s.e}\left(\bar{C}_{H C P}^{*}-\bar{C}_{M H C P}^{*}\right) \\
\leq C_{H C P}^{*}-C_{M H C P}^{*} \leq \\
\bar{C}_{H C P}^{*}-\bar{C}_{M H C P}^{*}+t_{\alpha / 2, n-1} \operatorname{see}\left(\bar{C}_{H C P}^{*}-\bar{C}_{M H C P}^{*}\right)
\end{gathered}
$$

where:

$t_{\alpha / 2, n-1}$ is the student coefficient function of $n$ and $\alpha$, with $n$ the number of replications (set at 10) and (1- $\alpha$ ), the confidence level (set at 95\%).

$\operatorname{s.e}\left(\bar{C}_{H C P}^{*}-\bar{C}_{M H C P}^{*}\right)=\frac{S_{D}}{\sqrt{n}}$ Standard error, $S_{D}^{2}=\frac{1}{n-1}\left(\sum_{i=1}^{n}\left(C_{H C P i}^{*}-C_{M H C P i}^{*}\right)^{2}-n\left(\bar{C}_{H C P}^{*}-\bar{C}_{M H C P}^{*}\right)^{2}\right)$

$\bar{C}_{H C P}^{*}$ the average optimal cost incurred under HCP.

$\bar{C}_{M H C P}^{*}$ the average optimal cost incurred under MHCP

The two configurations under study (HCP and MHCP) were simulated with their optimal design parameters, and the results are presented in Table 7.

Table 7: MHCP vs. HCP incurred costs for cases 1 to 5, $95 \%$ confidence interval

\begin{tabular}{||c|c|c|c|c|c|c||}
\hline & CASE & 1 & 2 & 3 & 4 & 5 \\
\hline MHCP & $C_{M H C P}^{*}$ & 83 & 90 & 112 & 119 & 125 \\
\hline HCP & $C_{H C P}^{*}$ & 89.4 & 98.6 & 114 & 123.4 & 130 \\
\hline \multirow{2}{*}{$\begin{array}{l}\text { Confidence } \\
\text { interval }\end{array}$} & Lower bound & 0.5 & 1.72 & 1 & 1.6 & 0.5 \\
\cline { 2 - 7 } & Upper bound & 0.95 & 2.3 & 1.5 & 3.2 & 1.7 \\
\hline
\end{tabular}


It has been shown that in all cases, it can be concluded that $C_{H C P}^{*}-C_{M H C P}^{*}>0$ at the $95 \%$ confidence level. Consequently, the MHCP gives the lower optimal cost, and furthermore, it appears that the MHCP is better than the HCP, and can be used to better approximate the optimal control policy.

\subsection{Usefulness of the MHCP}

As mentioned in the preceding sections, the production and setup policies given by equations (9) to (12) are completely defined for the given values of $a_{i}$ and $Z_{i}(\mathrm{i}=1,2)$. These equations explicitly stipulate a feedback policy, and based on the stock levels and the state of the machine, specify the best action to be taken so as to minimize the expected total discounted cost. Thus, the production policy states that when the machine is set up for a one-part type $i$, production must proceed at the maximum rate until the hedging level $Z_{i}$ is reached, and then proceed at the demand rate at $Z_{i}$ and stop beyond $Z_{i}$. This policy is conditional on the setup policy. It states that when $a_{i} \leq X_{i}$ and $X_{j}<0$, a setup action must be performed from product type $i$ to product type $j$.

In what follows (Figure 9), the quantified feedback policy of the basic case of the sensitivity analysis (Table 5, section 6.4) is presented. This illustration shows the actions that should be taken when the machine is producing part type 1, and is a function of the stock level of product type 1 and type $2\left(X_{1}\right.$ and $\left.X_{2}\right)$. When the machine is producing part type 2, a mirror schema could be realized so as to achieve a complete production and setup strategy. 


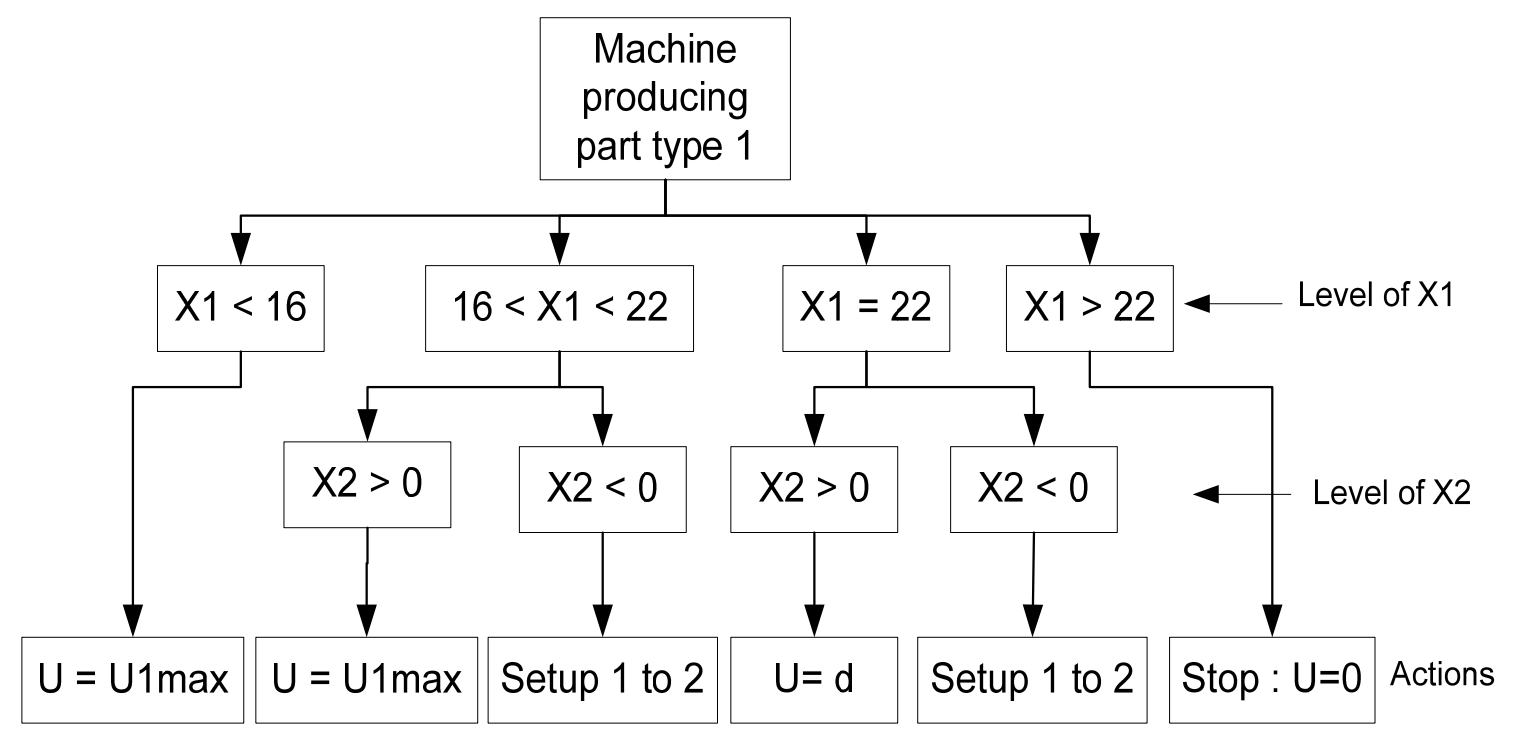

Figure 9: Practical solution

\section{Extension to five-machine manufacturing system with Non- exponential failure and repair time distributions}

Within the framework of the classical control theory of the last 30 years, no satisfactory method has been devised for the stochastic optimal control of manufacturing systems subject to non-exponential machine up and down times. In fact, the exponential distribution is used to develop optimality conditions, as shown in section 2. With nonexponential failure and repair time distributions and/or random demand rates, optimality conditions such as those given by equations (7) are very difficult to develop. However, that is the situation that is usually encountered in real manufacturing systems. We refer the reader to Law and Kelton (2000), chapter 6, for details on commonly used demand rates or failure and repair probability time distributions.

Despite the demand fluctuations and the types of failure and repair time distributions, a near-optimal control policy could be determined for an unreliable multiple-machine multiple-part type manufacturing system, in a much more complex situation (with nonexponential up and down distribution times for machines and/or random demand rates for products).

In the next paragraph, we will explain how this can be done, based on our previous works. In fact, we have already extended the concept of the hedging point policy in the case of one machine producing one kind of product with non-exponential failure and 
repair time distributions (Kenné and Gharbi (2000)) and in the case of multiple-product multiple-machine manufacturing systems not involving setups (Gharbi and Kenné, (2003)).

For the type of manufacturing system presented in this paper, an exponential failure and repair time distributions and a constant demand rate are first used in order to allow the definition of the optimal setup and production policies, which are then described through a modified hedging corridor policy (MHCP). The structure of such a policy is then parameterized by factors representing the thresholds of the products involved and the boundaries of the corridors. To quantify such a policy, simulation experiments are combined with experimental design and response surface methodology to estimate the optimal values of the MHCP's parameters. In the case of non-exponential machine up and down times and/or random demand rates, the quantification of the MHCP's parameters is possible with the help of the simulation model, which can easily take into account the nature of the machine's failure and repair time distributions and the randomness of the demand. The corresponding output is then given by the simulation model (i.e., cost) which affects the response surface model.

Let us now develop the quantified control policy for the five-machine two-product manufacturing systems case. The studied system is subject to non-exponential failure and repair time distribution (i.e., lognormal distribution for example).

Table 8 presents the data parameters of two cases (i.e., identical and different machine) describing the five-machine two-product manufacturing system under study. As mentioned previously, the numerical methods for such a system are characterized by a large dimension (see section 3.1). In addition, we consider in this section non-exponential distribution machines running and down times. In such situation the system is known as complex one 
Table 8: Data table for five-machine two-product manufacturing system

\begin{tabular}{|c|c|c|c|c|c|c|c|c|}
\hline Parameters & $\begin{array}{c}\left(u_{1 j}^{\max }, u_{2 j}^{\max }\right) \\
, \mathrm{j}=1, \ldots, 5\end{array}$ & $\left(d_{1}, d_{2}\right)$ & $\begin{array}{l}\left(\Theta_{12}^{j}, \Theta_{21}^{j}\right) \\
, j=1, \ldots, 5\end{array}$ & \multicolumn{3}{|c|}{ MTBF } & \multicolumn{2}{|c|}{ MTTR } \\
\hline $\begin{array}{c}\text { Case1 } \\
\text { Identical } \\
\text { machines }\end{array}$ & $(1,1)$ & $(2,2)$ & $(1,1)$ & \multicolumn{3}{|c|}{$\begin{array}{c}\text { Lognormal } \\
\quad(95,10)\end{array}$} & \multicolumn{2}{|c|}{$\begin{array}{c}\text { Lognormal } \\
(2.5,1.5)\end{array}$} \\
\hline \multirow{7}{*}{$\begin{array}{c}\text { Case } 2 \\
\text { Different } \\
\text { machines }\end{array}$} & \multirow{7}{*}{$(1,1)$} & \multirow{7}{*}{$(2,2)$} & \multirow{7}{*}{$(1,1)$} & \multicolumn{3}{|c|}{ Lognormal } & \multicolumn{2}{|c|}{ Lognormal } \\
\hline & & & & & $\mu$ & $\sigma$ & $\mu$ & $\sigma$ \\
\hline & & & & 1 & 100 & 10 & 2.6 & 1.5 \\
\hline & & & & 2 & 90 & 10 & 2.7 & 1.5 \\
\hline & & & & 3 & 85 & 10 & 2.3 & 1.5 \\
\hline & & & & 4 & 105 & 10 & 2.4 & 1.5 \\
\hline & & & & 5 & 95 & 10 & 2.5 & 1.5 \\
\hline
\end{tabular}

We present in Table 9 and Table 10 the incurred optimal costs for the same sensitivity analysis input, conducted for the one-machine two- product manufacturing system. It is important to note that the results presented in Table 9 and Table 10 under the HCP and MHCP policies, respectively, were obtained under the same conditions (simulation, experimental design and RSM).

Table 9: Optimal design factors and incurred costs with HCP

\begin{tabular}{|c|c|c|c|c|c|c|c|c|c|}
\hline Cases & $K_{i j}^{j}$ & $c_{1}^{+}$ & $c_{1}^{-}$ & $c_{2}^{+}$ & $c_{2}^{-}$ & $Z^{*}($ case 1$)$ & $c_{H C P}^{*}($ case 1$)$ & $Z^{*}$ (case2) & $c_{H C P}^{*}($ case2) \\
\hline 1 & 6 & 5 & 8 & 5 & 8 & 6 & 68 & 7 & 68.5 \\
\hline 2 & 6 & 5 & 10 & 5 & 10 & 7 & 72 & 8 & 72.2 \\
\hline 3 & 6 & 5 & 15 & 5 & 15 & 8 & 78 & 9 & 78.3 \\
\hline 4 & 6 & 5 & 20 & 5 & 20 & 9 & 82 & 10 & 82 \\
\hline 5 & 6 & 5 & 25 & 5 & 25 & 9 & 84 & 10 & 84.6 \\
\hline
\end{tabular}

The results obtained show that under HCP, the variation of the design parameter for the two cases (i.e., 1 and 2) does make sense (see section 6.4 and 6.5 for details). In addition, the incurred costs for all the cases are higher than those incurred under MHCP. This 
result is shown in Table 10. In the same direction, we observe that the incurred costs (under HCP or MHCP) for the five machine case (see Table 9 and 10) are lower than those for the one machine case (see table 5 and 6 ). This observation is explained by the fact that in the five parallel machines case, the system has more capacity than does an equivalent system with only one machine (i.e., the machines do not fall down simultaneously).

Table 10: Optimal design factors and incurred costs with MHCP

\begin{tabular}{|c|c|c|c|c|c|c|c|c|c|}
\hline Cases & $K_{i j}$ & $c_{1}^{+}$ & $c_{1}^{-}$ & $c_{2}^{+}$ & $c_{2}^{-}$ & $\begin{array}{c}\left(\mathrm{a}^{*}, \mathrm{Z}^{*}\right) \\
\text { Case1 }\end{array}$ & $\begin{array}{c}c_{M H C P}^{*} \\
\text { Case1 }\end{array}$ & $\begin{array}{c}\left(\mathrm{a}^{*}, \mathrm{Z}^{*}\right) \\
\text { Case 2 }\end{array}$ & $\begin{array}{c}c_{M H C P}^{*} \\
\text { Case 2 }\end{array}$ \\
\hline 1 & 6 & 5 & 8 & 5 & 8 & $(3,10)$ & 54 & $(4,11)$ & 54.87 \\
\hline 2 & 6 & 5 & 10 & 5 & 10 & $(4,11)$ & 61 & $(5,12)$ & 61.2 \\
\hline 3 & 6 & 5 & 15 & 5 & 15 & $(6,12)$ & 72 & $(7,13)$ & 72.43 \\
\hline 4 & 6 & 5 & 20 & 5 & 20 & $(7,13)$ & 80 & $(8,14)$ & 80.5 \\
\hline 5 & 6 & 5 & 25 & 5 & 25 & $(8,14)$ & 82 & $(9,15)$ & 82.5 \\
\hline
\end{tabular}

Following the same approach than section 6.5 , it can be concluded that in all cases $C_{H C P}^{*}-C_{M H C P}^{*}>0$ at the $95 \%$ confidence level. Consequently, the MHCP gives the lower optimal cost, and furthermore, it appears that the MHCP is better than the HCP, and can be used to better approximate the optimal control policy.

\section{Conclusion}

In this paper, we studied the production and setup control problem for an unreliable multiple-machine multiple-part type manufacturing system and solved the problem in the case of two products. We adopted a numerical approach for solving the HJB equations of the problem, and obtained near-optimal production and setup control policies. The optimal setup policy has been shown in this paper to be described by a Modified Hedging Corridor policy. Based on the numerical solution obtained, a parameterized near-optimal control policy was derived. Such a policy depends on the stock threshold levels and the boundaries of the corridor. To determine the parameters of the control policy, and hence, to achieve a close approximation of the optimal production and setup policies, an 
experimental approach based on design of experiment, simulation modeling and response surface methodology has been presented.

The proposed approach shows that the optimal cost incurred under the developed control policy is lower than that incurred under the hedging corridor policy. Moreover, the proposed combined approach offers an easily applied procedure to control manufacturing systems at the operational level. Based on the parameterized control policy obtained for the one machine two-product manufacturing system case, we presented the extension to the five-machine two-product case subject to non-exponential failure and repair time distributions.

\section{References}

AKELLA, R. and KUMAR, P. R., 1986, Optimal control of production rate in a failureprone manufacturing system. IEEE Transactions on Automatic Control, AC-31, 116-126.

BOUKAS, E. K. and KENNÉ, J. P., 1997, Maintenance and production control of manufacturing systems with setups. Lectures in applied mathematics (LAM), 33, $55-70$.

BAI, S. X. and ELHAFSI, M., 1997, Scheduling of an unreliable manufacturing system with non-resumable setups. Computers and Industrial Engineering, 32(4): 909-925.

GHARBI, A. and KENNÉ, J. P., 2003, Optimal production control problem in stochastic multiple-product multiple-machine manufacturing systems. IIE Transactions, 35, 941-952.

GHARBI, A. and KENNÉ, J. P., 2000, Production and preventive maintenance rates control for a manufacturing system: an experimental design approach. International Journal of Production Economics, 65(3), 275-287.

HAJJI, A., GHARBI, A. and KENNÉ, J. P., 2004, production and setup control of a failure prone manufacturing system, International Journal of Production Research, 42(6), 1107-1130.

KENNÉ J.P. and GHARBI, A., 2000, A Production Planning Problem in Manufacturing systems with General Failure and Repair Times Distributions, Production Planning \& Control Journal, 11(6), 581-587. 
KENNÉ, J. P. and GHARBI, A., 1999, Experimental design in production and maintenance control problem of a single machine, single product manufacturing system. International Journal of Production Research, 37(3), 621-637

KIMEMIA, J. G. and GERSHWIN, S. B., 1983, An algorithm for the computer control production in flexible manufacturing systems. IEEE Transactions, AC-15, 353-362.

KUSHNER, H. J. and DUPUIS, P. G., 1992, Numerical methods for stochastic control problems in continuous time (New York: Springer-Verlag).

LIBEROPOULOS, G. and CARAMANIS, M., 1997, Numerical investigation of optimal policies for production flow control and set-up scheduling: lessons from two-parttype failure-prone FMSs. International Journal of Production Research, 35(8), 2109-2133.

LAW, A. M. and KELTON, W. D., 2000, Simulation modeling and analysis, $\left(3^{\text {rd }}\right.$ edition, McGraw-Hill).

MONTGOMERY, D. C., 2001, Design and analysis of experiments, $\left(5^{\text {th }} \mathrm{edn}\right.$, John Wiley \& Sons).

PRITSKER, A. A. B. and O'REILlY, J. J., 1999, Simulation with Visual SLAM and Awesim, John Wiley \& Sons.

SETHI, S. P. and ZHANG, H., 1999, Average-Cost Optimal Policies for an Unreliable Flexible Multiproduct Machine. The International Journal of Flexible Manufacturing Systems, 11, 147-157.

SETHI, S. P. and ZHANG, Q., 1994, Hierarchical decision making in stochastic manufacturing systems (Boston: Birkhauser).

YAN, H. and ZHANG, Q., 1997, A numerical method in optimal production and setup scheduling of stochastic manufacturing systems. IEEE Transactions on Automatic control, 42(10), 1452-1455. 